INPLASY

PROTOCOL

To cite: Wang et al. Traditional Chinese Medicine Constitution Correlated with Asthma: A

Systematic Review and MetaAnalysis. Inplasy protocol 202180087. doi:

10.37766/inplasy2021.8.0087

Received: 23 August 2021

Published: 23 August 2021

Corresponding author: Ji Wang

doctorwang2009@126.com

Author Affiliation:

Center for Studies in

Constitution Research of

Traditional Chinese Medicine,

Beijing University of Chinese

Medicine.

Support: None.

Review Stage at time of this submission: The review has not yet started.

Conflicts of interest:

None declared.

\section{Traditional Chinese Medicine Constitution Correlated with Asthma: A Systematic Review and Meta- Analysis}

Wang, Y1; Liu, Z2; Wang, J3; Zhao, W4; Luo, B5; Wang, Y6.

Review question / Objective: The aim of this meta-analysis of randomized controlled trials is to explore the distribution of constitution types of asthma in traditional Chinese medicine (TCM) and to provide evidence-based medicine basis for the prevention and treatment of asthma.

Eligibility criteria: Inclusion: All clinical literature (including cross-sectional studies, case-control studies, and cohort studies) on the relationship between asthma and TCM constitution types are included, and there are no restrictions on the type of research and the form of publication. The details are as follows: (1) Research object: the research object is the asthma patients with a definite diagnosis; (2) Research tool: the measurement tool used for the constitution identification of the research object is the standard of Classification and Judgment of TCM Constitution issued by the China Institute of TCM in 2009; and (3) the sample size of the study is clear, and the data of the constitution composition are complete.Exclusion: (1) literature lacking in basic information reports or without statistics of constitution composition; (2) included subjects combined with other systematic serious diseases that may affect their TCM constitution types; (3) the study is limited to a certain kind of constitution population; and (4) Documents published multiple times with the same data.

INPLASY registration number: This protocol was registered with the International Platform of Registered Systematic Review and Meta-Analysis Protocols (INPLASY) on 23 August 2021 and was last updated on 23 August 2021 (registration number INPLASY202180087).

\section{INTRODUCTION}

Review question / Objective: The aim of this meta-analysis of randomized controlled trials is to explore the distribution of constitution types of asthma in traditional Chinese medicine (TCM) and 
to provide evidence-based medicine basis for the prevention and treatment of asthma.

Condition being studied: Bronchial asthma (asthma) is a chronic inflammatory disease of the airway involving a variety of cells and cell components. It is one of the common diseases of the respiratory system. Its clinical manifestations are often recurrent wheezing, shortness of breath, chest tightness or coughing symptoms, which worsen at night or in the early morning. According to statistics, there are about $\mathbf{3 0 0}$ million people with asthma in the world and more than $\mathbf{4 0}$ million people with asthma in China. The number of deaths due to asthma each year accounts for $1 / 250$ of the global death rate. Because asthma is lingering and difficult to cure repeatedly, Western medicine has no cure plan, which brings a heavy burden to patients' health and economic conditions. Therefore, it has important social value to explore good strategies for treating asthma in Chinese medicine. TCM constitution is an important part of TCM theory. It uses physique identification as methods and means to comprehensively improve people's health. The constitution of traditional Chinese medicine believes that the type of constitution affects the susceptibility and tendency of diseases. In recent years, research on the relationship between asthma and TCM constitution has gradually become a hot spot in clinical research, but there is no systematic review and analysis yet. The purpose of this study is to conduct a systematic review and meta-analysis of existing clinical research on the association between asthma patients and TCM constitution types, to obtain a larger sample of data on the distribution of TCM constitution types of asthma patients, and to dig deeper into asthma and TCM constitution from multiple factors The internal relevance of, provides a strong evidence-based medicine basis for clinical and scientific research.

\section{METHODS}

Participant or population: Asthma patients.

Intervention: None.
Comparator: Healthy people.

Study designs to be included: Crosssectional study, Cohort study, Case-control study.

Eligibility criteria: Inclusion: All clinical literature (including cross-sectional studies, case-control studies, and cohort studies) on the relationship between asthma and TCM constitution types are included, and there are no restrictions on the type of research and the form of publication.The details are as follows: (1) Research object: the research object is the asthma patients with a definite diagnosis; (2) Research tool: the measurement tool used for the constitution identification of the research object is the standard of Classification and Judgment of TCM Constitution issued by the China Institute of TCM in 2009; and (3) the sample size of the study is clear, and the data of the constitution composition are complete.Exclusion: (1) literature lacking in basic information reports or without statistics of constitution composition; (2) included subjects combined with other systematic serious diseases that may affect their TCM constitution types; (3) the study is limited to a certain kind of constitution population; and (4) Documents published multiple times with the same data.

Information sources: PubMed, Embase, Web of Science, China National Knowledge Infrastructure (CNKI), VIP Database (VIP), and Wanfang Database.

Main outcome(s): Through data analysis, the individual incidence of different TCM constitution types in asthma patients can be obtained, and the results are expressed in percentages and their $95 \%$ confidence interval (Cl). When the heterogeneity is large, a subgroup analysis of age and gender is performed. For the results of the case control group, the odds ratio (OR) and its $95 \% \mathrm{Cl}$ were used to describe the effect size of a single study. According to the heterogeneity selection effect model, the odds ratio (OR) between asthma patients 
and the general population with a certain physique as an exposure factor was obtained.

Quality assessment / Risk of bias analysis: Each paper is screened and extracted by two investigators independently, and the results are cross-checked in every step. In the case of divergence in cross-check, the checked paper will be examined by the 3rd investigator for the final decision. The Unit States Agency for Healthcare Research (AHRQ) publishes standards to evaluate cross-sectional study. As for the mark, 0-3 is considered as low quality, 4-7 as medium quality, and $8-11$ as high quality. The Newcastle-Ottawa scale (NOS) recommends case-control study and cohort study. Its highest mark is 9, and any mark over 6 is considered as high quality. Results from these questions will be graphed and assessed using Stata 16.0.

Strategy of data synthesis: The metaanalysis of the individual rate of each constitution type was carried out by using STATA 16.0. The analysis model is selected according to the heterogeneity test results. Obtain the percentage of different constitution types of asthma patients and their $95 \%$ confidence interval $(\mathrm{Cl})$. When the heterogeneity was too large, subgroup analysis was carried out according to regional and age factors, and the inverted funnel chart was used to analyze the degree of publication bias assessed by funnel plot analysis. Statistical analysis of two groups of patients with STATA16.0, the odds ratio (OR) and its $95 \% \mathrm{Cl}$ were used to describe the effect value of a single study. The effect model was selected according to heterogeneity: the fixed effect model was used when heterogeneity was less than $50 \%$, and the random effect model was used when heterogeneity was greater $(12>50 \%)$.

Subgroup analysis: We will consider subgroups such as region, gender, and related factors.

Sensitivity analysis: Judge the degree of heterogeneity through the results of 12 and
$P$ values, and choose the effect model: when the heterogeneity is small (I 20.1), the fixed-effect model is used; otherwise, the heterogeneity is large. Random effects model. And get the result of "incidence rate" and the range of $95 \% \mathrm{Cl}$.

Country(ies) involved: China.

Keywords: Asthma; constitution; Chinese medicine.

Contributions of each author:

Author 1 - Yaqi WANG.

Author 2 - Zhidan LIU.

Author 3 - Ji WANG.

Author 4 - Weibo ZHAO.

Author 5 - Bin LUO.

Author 6 - Yixing WANG. 